\title{
Unusual Fluorescent Responses of Morpholine-functionalized Fluorescent Probes to pH via Manipulation of BODIPY's HOMO and LUMO Energy Orbitals for Intracellular pH Detection
}

\author{
Jingtuo Zhang ${ }^{\dagger}$, Mu Yang ${ }^{\dagger}$, Wafa Mazi ${ }^{\dagger}$, Kapil Adhikari ${ }^{\dagger}$, Mingxi Fang ${ }^{\dagger}$, Fei Xie ${ }^{\dagger}$, Loredana \\ Valenzano ${ }^{\dagger,}$, , Ashutosh Tiwari ${ }^{\dagger,}$, , Fen-Tair Luo ${ }^{\ddagger}$, , and Haiying Liu ${ }^{\dagger,}{ }^{*}$ \\ † Department of Chemistry, Michigan Technological University, Houghton, MI 49931 \\ ‡ Institute of Chemistry, Academia Sinica, Taipei, Taiwan 11529, Republic of China
}

\begin{abstract}
Three uncommon morpholine-based fluorescent probes (A, B and $\mathbf{C})$ for $\mathrm{pH}$ were prepared by introducing morpholine residues to BODIPY dyes at 4,4'- and 2,6-positions, respectively. In contrast to morpholine-based fluorescent probes for $\mathrm{pH}$ reported in literature, these fluorescent probes display high fluorescence in a basic condition while they exhibit very weak fluorescence in an acidic condition. The theoretical calculation confirmed that morpholine is unable to function as either an electron donor or an electron acceptor to quench the BODIPY fluorescence in the neutral and basic condition via photo-induced electron transfer (PET) mechanism because the LUMO energy of morpholine is higher than those of the BODIPY dyes while its HOMO energy is lower than those of the BODIPY dyes. However, the protonation of tertiary amines of the morpholine residues in an acidic environment leads to fluorescence quenching of the BODIPY dyes via d-PET mechanism. The fluorescence quenching is because the protonation effectively decreases the LUMO energy which locates between the HOMO and LUMO energies of the BODIPY dyes. Fluorescent probe $\mathbf{C}$ with deep-red emission has been successfully used to detect $\mathrm{pH}$ changes in mammalian cells.
\end{abstract}

\section{Keywords}

BODIPY dye; Morpholine moiety; Intracellular pH; Photoinduced electron transfer (PET)

\begin{abstract}
Intracellular $\mathrm{pH}\left(\mathrm{pH}_{\mathrm{i}}\right)$ functions to regulate many cellular behaviors such as cell volume regulation, vesicle trafficking, cellular metabolism, cell membrane polarity, cellular signaling, and cell activation, growth, proliferation, apoptosis, enzyme activity and protein degradation. The $\mathrm{pH}_{\mathrm{i}}$ inside cells may vary considerably among subcellular compartments
\end{abstract}

\footnotetext{
*Corresponding Authors Dr. Haiying Liu hyliu@mtu.edu. *Dr. Ashutosh Tiwari Tiwari@mtu.edu. *Dr. Loredana Valenzano lvalenza@mtu.edu. Dr. Fen-Tair Luo luoft@gate.sinica.edu.tw.

Supporting Information Available: The following files are available free of charge.

Morpholine_BODIPY_Dyes_intracellular_pH_SI.pdf: The detailed synthetic procedures, ${ }^{1} \mathrm{H}$-and ${ }^{13} \mathrm{C}-\mathrm{NMR}$ spectra of BODIPY dyes 4 and 6, fluorescent probes $\mathbf{A}, \mathbf{B}$ and $\mathbf{C}$, detailed supplemental optical spectra of fluorescent probes $\mathbf{A}, \mathbf{B}, \mathbf{C}$ and their precursors (BODIPY dyes 1, 4 and 6) at different $\mathrm{pH}$ and in the presence of different metal ions, MTS method and the fluorescence images of HUVEC-C cells with probe $\mathbf{C}$ at different concentrations are included. This material is available free of charge via the Internet at http://pubs.acs.org
} 
such as the cytosol, mitochondria, endoplasmic reticulum, endosome, lysosome, and nucleus. The $\mathrm{pH}_{\mathrm{i}}$ in a typical mammalian cell can range from 4.7 in lysosome to 8.0 in mitochondria. The lysosomal acidic environment activates enzymes to facilitate the degradation of proteins during cellular breakdown. Disruptive $\mathrm{pH}_{\mathrm{i}}$ variations in organelles can lead to dysfunction of the organelles that can result in pathophysiology in humans triggering cancer, stroke, and/or Alzheimer's disease. Therefore, it is very important to monitor $\mathrm{pH}$ changes inside living cells in order to investigate cellular functions that can provide insight into physiological and pathological processes. Fluorescence-based techniques such as fluorescence microscopy and flow cytometry with high-resolution and high-throughput analysis have been widely used to investigate intact subcellular $\mathrm{pH}$ and possess many technical and practical advantages over other methods because of their high sensitivity, operational simplicity, and unrivaled spatial and temporal resolution. Many fluorescent probes for $\mathrm{pH}$ have been developed. However, only a few of them have been successfully used to monitor lysosomal $\mathrm{pH}$ inside living cells. ${ }^{1-8}$ Most fluorescent probes for lysosomal $\mathrm{pH}$, including the commercial probes have used morpholine residues for their selective accumulation in acidic lysosomes through protonation of the tertiary amine groups in a cellular acidic environment. ${ }^{9}$ The fluorescence enhancement results from the suppression of photo-induced electron transfer (PET) of the tertiary amine attached to the probe. ${ }^{9}$ Our hypothesis was that fluorescent probes based on morpholine could display unusual responses to $\mathrm{pH}$ if we could effectively manipulate HOMO and LUMO energy orbitals of fluorophores so that the tertiary amines from morpholine residues were unable to serve as electron donors to quench the fluorophore fluorescence via a-PET ("a" denotes fluorophore serve as electron acceptor) mechanism while the protonated morpholine moieties can function as electron acceptor to quench the fluorescence of the fluorophore via d-PET ("d" denotes excited fluorophore serve as electron donor) mechanism. In this paper, we reported three uncommon morpholine based fluorescent probes (A, B and $\mathbf{C})$ to sense $\mathrm{pH}$ which were prepared by introducing morpholine residues to BODIPY dyes at 4,4'-positions and 2,6-positions, respectively (Figure 1). The fluorescent probes display high fluorescence in a basic condition. However, they exhibit very weak fluorescence in an acidic condition. The theoretical calculation showed that the LUMO energy of morpholine is higher than those of the BODIPY dyes while its HOMO energy is lower than those of the BODIPY dyes. As a result, morpholine is unable to serve as an electron donor or an electron acceptor to quench the BODIPY fluorescence in the neutral and basic conditions via PET mechanism. Furthermore, the protonation of tertiary amines of the morpholine residues in an acidic environment effectively decreases the LUMO energy so that it locates between the HOMO and LUMO energies of the BODIPY dyes, resulting in fluorescence quenching of the BODIPY dyes via d-PET mechanism. These probes also possesses great photostability and selectivity at different $\mathrm{pH}$ values. Among them, fluorescent probe $\mathbf{C}$ has more advantages for live cell fluorescence imaging because of its long emission wavelength, large Stokes shifts and high $\mathrm{p} K_{\mathrm{a}}$ near physiological $\mathrm{pH}$ and it displays turn-on fluorescence responses to the increasing intracellular $\mathrm{pH}$, demonstrating the potential applications for non-invasive monitoring $\mathrm{pH}$ changes inside of living cells. 


\section{Experimental section}

\section{Instrumentation}

${ }^{1} \mathrm{H}$ NMR and ${ }^{13} \mathrm{C}$ NMR spectra were collected by $400 \mathrm{MHz}$ Varian Unity Inova NMR spectrophotometer instrument. ${ }^{1} \mathrm{H}$ and ${ }^{13} \mathrm{C}$ NMR spectra were recorded in $\mathrm{CDCl}_{3}$ and DMSO- $d_{6}$ solutions. Chemical shifts $(\delta)$ are given in ppm with solvent residual peaks $\left({ }^{1} \mathrm{H}: \delta\right.$ 7.26 for $\mathrm{CDCl}_{3}, \delta 2.50$ for DMSO- $d_{6}$; ${ }^{13} \mathrm{C}: \delta 77.3$ for $\mathrm{CDCl}_{3}$ ) as internal standards. HRMS were measured with electrospray ionization (ESI) mass spectrometer. Absorption spectra were taken on a Perkin Elmer Lambda 35 UV/VIS spectrometer. Fluorescence spectra were recorded on a Jobin Yvon Fluoro-max-4 spectrofluorometer.

\section{Materials}

Unless specific indicated, all reagents and solvents were obtained from commercial suppliers and used without further purification. Compound $\mathbf{2}$ was prepared according to the reported literature ${ }^{10}$

Fluorescent probe A-1 ${ }^{1} \mathrm{H}$ NMR $\left(400 \mathrm{MHz}, \mathrm{CDCl}_{3}\right): \delta 6.99(\mathrm{~d}, J=8 \mathrm{~Hz}, 1 \mathrm{H}), 6.85(\mathrm{~d}, J$ $=2 \mathrm{~Hz}, 1 \mathrm{H}), 6.82(\mathrm{dd}, J=2,8 \mathrm{~Hz}, 1 \mathrm{H}), 6.00(\mathrm{~s}, 2 \mathrm{H}), 4.21(\mathrm{t}, J=5.2 \mathrm{~Hz}, 2 \mathrm{H}), 4.12(\mathrm{t}, J=5.2$ $\mathrm{Hz}, 2 \mathrm{H}), 3.91(\mathrm{t}, J=5.2 \mathrm{~Hz}, 2 \mathrm{H}), 3.86(\mathrm{t}, J=5.2 \mathrm{~Hz}, 2 \mathrm{H}), 3.79-3.51(\mathrm{~m}, 24 \mathrm{H}), 3.38(\mathrm{~s}$, $3 \mathrm{H}), 3.36(\mathrm{~s}, 3 \mathrm{H}), 3.28(\mathrm{~s}, 4 \mathrm{H}), 2.73(\mathrm{~s}, 6 \mathrm{H}), 2.56(\mathrm{br}, 8 \mathrm{H}), 1.45(\mathrm{~s}, 6 \mathrm{H}) .{ }^{13} \mathrm{C}$ NMR $(100$ $\left.\mathrm{MHz}, \mathrm{CDCl}_{3}\right): \delta 155.1,149.9,149.5,141.2,130.1,128.6,121.5,114.8,114.6,72.2,71.2$, 71.0, 70.9, 70.8, 70.0, 69.3, 69.0, 67.2, 59.3, 52.48, 7.8, 16.5, 15.0. HRMS (ESI): calculated for $\mathrm{C}_{47} \mathrm{H}_{67} \mathrm{BN}_{4} \mathrm{O}_{10} \mathrm{Na}[\mathrm{M}+\mathrm{Na}]$, 881.4842; found, 881.4832.

Fluorescent probe B- ${ }^{1} \mathrm{H} \mathrm{NMR}\left(400 \mathrm{MHz}, \mathrm{CDCl}_{3}\right): \delta 6.95(\mathrm{~d}, J=8 \mathrm{~Hz}, 1 \mathrm{H}), 3.59$ $3.55(\mathrm{~m}, 2 \mathrm{H}), 4.13(\mathrm{t}, J=4.8 \mathrm{~Hz}, 2 \mathrm{H}), 4.01(\mathrm{t}, J=4.8 \mathrm{~Hz}, 2 \mathrm{H}), 3.86(\mathrm{t}, J=4.8 \mathrm{~Hz}, 2 \mathrm{H}), 3.74$ - $3.28(\mathrm{~m}, 32 \mathrm{H}), 3.15(\mathrm{~s}, 4 \mathrm{H}), 2.52$ (s, 6H), 2.31 (br, $138 \mathrm{H}), 1.42$ (s, 6H). C NMR (100 $\left.\mathrm{MHz}, \mathrm{CDCl}_{3}\right)$ : $\delta 161.2,156.8,155.2,141.1,138.7,131.5,130.2,126.2,117.2,106.5,100.7$, 72.1, 72.0, 71.1, 71.0, 70.8, 70.7, 70.6, 70.4, 69.8, 69.2, 68.9, 67.8, 67.2, 59.2, 59.1, 53.5, 52.1, 12.9, 11.8. HRMS (ESI): calculated for $\mathrm{C}_{43} \mathrm{H}_{64} \mathrm{BF}_{2} \mathrm{~N}_{4} \mathrm{O}_{10}[\mathrm{M}-\mathrm{H}]^{-}, 845.4792$; found, 845.4809.

Fluorescent probe $\mathbf{C}-{ }^{1} \mathrm{H}$ NMR $(400 \mathrm{MHz}, \mathrm{CDCl}): \delta 7.18-7.09(\mathrm{~m}, 3 \mathrm{H}), 6.77(\mathrm{~d}, J=$ $8 \mathrm{~Hz}, 2 \mathrm{H}), 6.45$ (s, 1H), 5.90 (d, $J=16 \mathrm{~Hz}, 2 \mathrm{H}), 4.14(\mathrm{t}, J=5.2 \mathrm{~Hz}, 4 \mathrm{H}), 4.07$ (t, $J=5.2 \mathrm{~Hz}$, $4 \mathrm{H}), 3.90-3.40(\mathrm{~m}, 72 \mathrm{H}), 3.38(\mathrm{~s}, 3 \mathrm{H}), 3.37$ (s, 6H), $3.36(\mathrm{~s}, 3 \mathrm{H}), 3.34(\mathrm{~s}, 6 \mathrm{H}), 3.31(\mathrm{~s}, 3 \mathrm{H})$, 3.23(brs, 4H), 2.62 (s, 6H), 2.50 (s, 8H). ${ }^{13} \mathrm{C}$ NMR (100 MHz, CDCl ): $\delta$ 172.5, 161.3, 157.8, 149.0, 148.9, 146.2, 142.7, 135.0, 131.3, 129.2, 124.3, 120.1, 114.4, 111.9, 106.7, 100.3, 97.5, 71.9, 71.8, 71.0, 70.8, 70.7, 70.6, 70.5, 70.4, 70.2, 69.7, 68.8, 68.6, 58.9. HRMS (ESI): calculated for C H BF N O [M-H] ${ }^{-}, 1669.8987$; found, 1669.8971.

\section{Optical measurement}

$20 \mathrm{mM}$ citrate-phosphate-borate buffer was used for $\mathrm{pH}$ dependency and photostability measurements of fluorescent probes. To avoid the interference caused by metalphosphate and metal-citrate binding interactions (forming precipitates of divalent cation phosphate and 
forming complex of the metal-citrate), $10 \mathrm{mM} \mathrm{KHP} \mathrm{buffer} \mathrm{(pH} \mathrm{4.0)} \mathrm{and} 10 \mathrm{mM}$ HEPES (pH 7.4) buffer were used for selectivity measurements of fluorescent probes $\mathbf{A}, \mathbf{B}$ and $\mathbf{C}$.

\section{Computational modeling}

Electronic properties of the chemical structures of all the fluorescent probes were calculated using density functional theory (DFT) incorporating Hartree-Fock (HF) exchange with DFT exchange-correlation. In particular, the hybrid functional B3LYP ${ }^{11-13}$ and all electron basis sets $6-311 \mathrm{G}(2 \mathrm{~d}, 2 \mathrm{p})^{14}$ as implemented in the Gaussian09 suite of programs ${ }^{15}$ were used for full geometry optimization of the probes. HOMO and LUMO were calculated for the optimized geometries. All calculations were performed in vacuum.

\section{Live cell fluorescence imaging}

Normal endothelial cells HUVEC-C (from ATCC) were plated on 12-well culture plates at 1 $\times 10^{5}$ cells/well and were incubated at $37{ }^{\circ} \mathrm{C}$ in $5 \% \mathrm{CO}_{2}$ incubator overnight. Next day, the medium was removed and cells were rinsed twice with $1 \mathrm{x}$ PBS (pH 7.4) followed by incubation with probe $\mathbf{C}$ for $2 \mathrm{~h}$ at $5 \mu \mathrm{M}, 15 \mu \mathrm{M}$, or $25 \mu \mathrm{M}$ concentrations in fresh serum free media containing $1 \mu \mathrm{M}$ LysoSensor Green DND-189 (Life Technologies). Hoechst 33342 (Sigma-Aldrich) dye was added at $1 \mu \mathrm{g} \cdot \mathrm{mL}^{-1}$ and the cells were further incubated for 10 minutes. After incubation, the medium was removed, cells were gently rinsed with PBS (pH 7.4) three times. The cells were treated with nigericin $\left(5 \mu \mathrm{g} \cdot \mathrm{mL}^{-1}\right)$ in potassium rich PBS with pH range from 5.5, 6.5, 7.5 to 8.5, and incubated further for 15 min. Live cell images were acquired using an inverted fluorescence microscope (AMF-4306, EVOSfl, AMG) with DAPI filter for Hoechst 33342 (Sigma-Aldich), GFP filter for LysoSensor Green, and RFP filter for fluorescent probe $\mathbf{C}$. The fluorescence images were obtained at $40 \times$ magnification. The exposure times for each filter were kept constant. Co-localization analysis based on Pearson's coefficient was done using JACoP plugin from ImageJ. ${ }^{16}$

\section{Results and discussion}

\section{Synthetic approach}

In order to prepare morpholine-functionalized BODIPY-based fluorescent probes for $\mathrm{pH}$, we incorporated morpholine moieties onto the BODIPY core at 4,4'-positions and 2,6-positions while we introduced ortho-or meta-substituent group of tri(ethylene glycol)methyl ether on the meso-phenyl rings and at 1,7-positions of BODIPY dyes to enhance their hydrophilicity and solubility in polar solvents (Scheme 1). Fluorescent probe $\mathbf{A}$ was prepared by replacing fluorine atoms of BOIDPY dye $\mathbf{1}$ at 4,4'-positions with 4-prop-2-ynyl-morpholine (2) (BODIPY dye 1 was synthesized according our reported procedures ${ }^{17}$ via a Grignard reaction. In order to incorporate morpholine moieties onto 2,6-positions of BODIPY core, formyl groups were introduced to the BODIPY dye $\mathbf{3}$ (it was also synthesized according to our reported procedures) ${ }^{18-19}$ at 2,6-positions via two-step Vilsmeier-Haack reactions, affording 2,6-diformyl BODIPY dye $\mathbf{4}$. Then a reductive amination of BODIPY dye $\mathbf{4}$ with morpholine was carried out by using sodium acetoxyborohydride to yield fluorescent probe B. To tune the BOIDPY core of fluorescence probe to longer emission wavelengths with smaller HOMO-LUMO energy gap, a Knoevenagel condensation of BODIPY dye $\mathbf{4}$ with benzaldehyde derivative (5) was conducted. The presence of formyl groups of BODIPY dye 
4 at 2,6-positions initiated formation of distyryl bonds at 1,7-positions instead of 3,5positions, yielding 2,6-diformyl-1,7-distyryl-BODIPY dye (6), which was confirmed by the clear disappearance of 1,7-methyl proton peak at $1.85 \mathrm{ppm}$ and the preservation of 3,5methyl proton peak at $2.92 \mathrm{ppm}$ in ${ }^{1} \mathrm{H}$ NMR spectrum of BODIPY dye 6 (please see Figures $\mathrm{S} 3$ and $\mathrm{S} 7$ in supporting information). This is because the 1,7-methyl groups become activated by introduction of formyl groups at 2,6-positions to BODIPY core, which has been discussed in our previous paper. ${ }^{17}$ 1,7-distyryl BODIPY dye bearing morpholine groups at 2,6-positions (fluorescent probe $\mathbf{C}$ ) was obtained by amination of BODIPY dye $\mathbf{6}$ with morpholine using sodium acetoxyborohydride in the same approach to prepare fluorescent probe $\mathbf{B}$. Fluorescent probes $\mathbf{A}, \mathbf{B}$ and $\mathbf{C}$ are easily dissolved in common organic solvents such as dichloromethane, ethanol, THF, DMF and DMSO. They can be readily dissolved in aqueous solution with the help of small amount of polar organic solvent as the polar cosolvent such as DMSO because of the hydrophilic features of tri(ethylene glycol)methyl ether residues and morpholine residues on the BODIPY cores.

\section{Optical properties}

Fluorescent probe A displays absorption and emission peaks of BODIPY dyes at $498 \mathrm{~nm}$ and $508 \mathrm{~nm}$ in ethanol, respectively. The fluorescence quantum yield of probe $\mathbf{A}$ in ethanol is 56\%. Fluorescent probe $\mathbf{B}$ shows absorption and emission peaks at 515 and $529 \mathrm{~nm}$ with a fluorescence quantum yield of $8 \%$, and exhibits $17 \mathrm{~nm}$ and $21 \mathrm{~nm}$ red shifts in absorption and emission spectra in ethanol, respectively, compared with fluorescent probe $\mathbf{A}$. The red shifts in absorption and emission peaks of fluorescent probe $\mathbf{B}$ may be due to the enhanced electron donating effect of methylene groups with adjacent electrondonating tertiary amines from the morpholine groups at 2,6-positions to the BODIPY core compared with methyl groups of fluorescent probe $\mathbf{A}$ at 2,6-positions, resulting in a decreased HOMO-LUMO energy gap. Compared with fluorescent probes $\mathbf{A}$ and $\mathbf{B}$, the significant red shifts in both absorption and emission spectra were observed for fluorescent probe $\mathbf{C}$ with absorption and emission peaks at 565 and $652 \mathrm{~nm}$ in ethanol solution, respectively, because of its significantly enhanced $\pi$-conjugation via its distyryl groups at 1,7 -positions. In addition, we examined the optical properties of fluorescent probes $\mathbf{A}, \mathbf{B}$ and $\mathbf{C}$ in aqueous solution at physiological pH (with 1\% DMSO as co-solvent). Fluorescent probes A and $\mathbf{B}$ show similar absorption and emission bands in aqueous solution with negligible shifts compared with those in ethanol while fluorescent probe $\mathbf{C}$, with an enlarged Stokes shifts of $115 \mathrm{~nm}$ in aqueous solution which is $28 \mathrm{~nm}$ larger than its Stokes shift of $87 \mathrm{~nm}$ in ethanol, was interestingly observed with $15 \mathrm{~nm}$ blue shift in absorption peak and $13 \mathrm{~nm}$ red shift in emission peak relative to its absorption and emission peaks in ethanol (see Table S1 in supporting information). The fluorescence quantum yields of the fluorescent probes $\mathbf{A}, \mathbf{B}$ and $\mathbf{C}$ significantly dropped from ethanol to buffer solution ( $\mathrm{pH}$ 7.4) as fluorescent probes show fluorescence quantum yields of $18 \%, 1.3 \%$ and $0.32 \%$ in buffer solution compared with those of $56 \%, 8 \%$ and $8.6 \%$ in ethanol solution, respectively. The low fluorescence quantum yields of the fluorescent probes in aqueous solution may result from fluorescence self-quenching due to the potential dye aggregations in aqueous solution. 


\section{Optical responses of fluorescent probes to $\mathrm{pH}$}

We investigated the $\mathrm{pH}$ effect of fluorescent probes $\mathbf{A}, \mathbf{B}, \mathbf{C}$, and their intermediates (BODIPY dyes 1, 4 and 6) in buffer solution. There were no obvious changes observed in both absorption and emission spectra of all intermediates $\mathbf{1 , 4}$ and $\mathbf{6}$ in different $\mathrm{pH}$ conditions (see Figure S11 in supporting information). However, fluorescent probes A, B and $\mathbf{C}$ exhibit very sensitive fluorescent responses to $\mathrm{pH}$. They show very low fluorescence in acidic condition while most morpholine-functionalized fluorescent probes reported in the literature are highly fluorescent in acidic condition because of the prohibited a-PET effect from morpholine moiety to the fluorophore through the protonation of morpholine moiety. However, change of the $\mathrm{pH}$ from an acidic condition of 3.02 to a basic condition of 9.49 results in significant increases of fluorescence intensity of fluorescent probes $\mathbf{A}, \mathbf{B}$ and $\mathbf{C}$ with 2.4-fold, 15.7-fold and 14.3-fold enhancements, respectively (Figure 2). This unique $\mathrm{pH}$-dependent behavior of the probes is in agreement with our hypothesis that protonated morpholine moieties can function as electron acceptors to quench the fluorescence of the BODIPY dye via d-PET mechanism while the tertiary amines from morpholine residues are unable to serve as electron donors to quench the BODIPY dye fluorescence via a-PET mechanism when the HOMO and LUMO energy orbitals of the BODIPY dye are controlled in a certain range. Fluorescent probe $\mathbf{A}$ shows a significant fluorescence intensity change between $\mathrm{pH} 4.0$ and $\mathrm{pH} 6.2$ with a $K_{\mathrm{a}}$ of 5.04 while the significant fluorescence changes of fluorescent probes $\mathbf{B}$ and $\mathbf{C}$ shift to the window between $\mathrm{pH} 4.5$ to $\mathrm{pH} 7.4$ with $\mathrm{p} K_{\mathrm{a}}$ of 6.16 and 6.15 , respectively (Figure 2). The different $\mathrm{p} K_{\mathrm{a}}$ values indicate the different availability (or basicity) of the nitrogen lone-pair electrons on the morpholine moieties of the probes for protonation, where fluorescent probe A possesses the least availability due to the electronwithdrawing property of the alkyne groups adjacent to the morpholine moieties. In addition, 11-nm and 10-nm blue shifts in fluorescence and absorption spectra were observed for probe B, respectively, when $\mathrm{pH}$ decreases from 9.49 to 3.02. The blue shifts may arise from weaker electron donating effect of methylene groups with adjacent electron-withdrawing protonated tertiary amines from morpholine moieties at 2,6-positions in an acid condition. The similar blue shifts (13 nm and $16 \mathrm{~nm}$ shifts in fluorescence and absorption, respectively) of probe $\mathbf{C}$ were also observed due to the same weaker electron-donating effect of methylene groups at 2,6-positions in an acid condition. In addition, the absorbance of probes $\mathbf{A}, \mathbf{B}$ and C at $470 \mathrm{~nm}, 480 \mathrm{~nm}$ and $535 \mathrm{~nm}$ respectively, which were used as the excitation wavelengths for their corresponding fluorescence spectra, display negligible changes under different $\mathrm{pH}$ values, (Figure 2). This further indicates that the $\mathrm{pH}$ responses of the probes are mainly due to d-PET effect instead of the absorbance change.

\section{Theoretical modeling}

In order to further reveal the structural properties of the fluorescent probes, and more importantly, to verify the hypothesis that the fluorescent probes respond to $\mathrm{pH}$ via a modulation of d-PET effect from BODIPY core to protonated morpholine moieties at different $\mathrm{pH}$ conditions, we calculated the HOMOs and LUMOs of fluorescent probes $\mathbf{A}, \mathbf{B}$ and $\mathbf{C}, N$-methylmorpholine and protonated $N$-methylmorpholine using density functional theory (DFT-B3LYP/6-311G(2d,2p)). The obtained results are shown in Figure 3. The results show that fluorescent probe $\mathbf{B}$ has a slightly smaller HOMO-LUMO energy gap (2.97 
$\mathrm{eV})$ than that of fluorescent probe $\mathbf{A}(3.07 \mathrm{eV})$, indicating a mild electron donating effect of methylene groups with adjacent electron-donating tertiary amine groups to the BODIPY core at 2,6-positions. This is in accord with the spectra red shifts observed in absorption and emission spectra of fluorescent probe $\mathbf{B}$ relative to those of fluorescent probe $\mathbf{A}$ in a basic condition (Figure 2 and Table $\mathbf{S} 1$ in supporting information). Fluorescent probe $\mathbf{C}$ possesses the smallest energy gap $(2.39 \mathrm{eV})$ among three probes; the increasing in the HOMO and the decreasing in the LUMO arise from the enhanced $\pi$-conjugation occurring via the distryl groups on the BODIPY core at 1,7-positions. The density distributions of HOMO-and LUMO-electrons on the probes show that there are no $\pi$-electrons spared onto the morpholine moieties for any of the three probes (Figure 3), indicating that the morpholine moieties are isolated from $\pi$-conjugations of the BODIPY cores. We calculated the HOMO and LUMO energy of morpholine moieties before and after protonation using $\mathrm{N}$ methylmorpholine as a model. As we expected, the calculated HOMO energy of morpholine moieties is lower than those of all three probes while its LUMO energy is higher than those of all three probes. This leads to a prohibited a-PET effect from morpholine moiety to the probe core. However, the protonation of the morpholine moiety in acidic condition dramatically reduces its HOMO and LUMO energies so that the LUMO energy of protonated morpholine moiety lies between of the HOMO and LUMO energies of the probes. As a result, the protonated morpholine moiety is able to serve as an electron acceptor allowing for the electron transition be possible from the LUMO of the fluorescent probes A, $\mathbf{B}$ or $\mathbf{C}$ to the LUMO of protonated morpholine moiety, resulting in the fluorescence quenching of the probes via a d-PET effect.

\section{Selectivity and photostability}

Considering the potential binding events between amines and metal ions when applying the fluorescent probes in complex environments such as physiological condition, we further investigate selectivity of fluorescent probes to $\mathrm{pH}$ over other metal ions. The results showed that $5 \mu \mathrm{M}$ fluorescent probes $\mathbf{A}, \mathbf{B}$ and $\mathbf{C}$ display excellent selective responses to $\mathrm{pH}$ over other metal ions because there is no significant change observed in terms of fluorescence intensity of the probes in the absence and presence of different metal ions $(200 \mu \mathrm{M})$ such as $\mathrm{K}^{+}, \mathrm{Na}^{+}, \mathrm{Mg}^{2+}$ and $\mathrm{Ca}^{2+}, \mathrm{Cu}^{2+}, \mathrm{Zn}^{2+}, \mathrm{Ni}^{2+}, \mathrm{Mn}^{2+}, \mathrm{Co}^{2+}, \mathrm{Fe}^{2+}$ and $\mathrm{Fe}^{3+}$ in buffer solution at pH 4.0 and 7.4(Figure 4, upper row). We also studied the photostability of fluorescent probes $\mathbf{A}, \mathbf{B}$ and $\mathbf{C}$ in both acidic ( $\mathrm{pH} 4.0$ ) and near neutral conditions ( $\mathrm{pH}$ 7.4). Under 2hour excitation (with excitation wavelength of $470 \mathrm{~nm}, 490 \mathrm{~nm}$ and $580 \mathrm{~nm}$ for fluorescent probes A $\mathbf{B}$ and $\mathbf{C}$, respectively), fluorescent probe $\mathbf{A}$ showed an excellent photostability with less than $1 \%$ decrease of fluorescence intensity within 2 hours. Fluorescent probes $\mathbf{B}$ and $\mathbf{C}$ also showed very good photostability in $\mathrm{pH} 4.0$ as fluorescent probe $\mathbf{A}$. While in $\mathrm{pH}$ 7.4, the fluorescence intensity of fluorescent probe $\mathbf{B}$ decreased by $7.5 \%$ in the first hour and further decreased by $2 \%$ in the second hour under excitation and fluorescent probe $\mathbf{C}$ had a gradually decrease by $3 \%$ in fluorescence intensity within 2-hour excitation (Figure 4, lower row). 


\section{In-vitro cell imaging and intracellular pH detection}

In order to determine if the fluorescent probes could selectively detect change in intracellular $\mathrm{pH}$ of live cells, we chose the fluorescent probe $\mathbf{C}$ for live cell fluorescence imaging with normal endothelial (HUVEC-C) cells at different intracellular $\mathrm{pH}$ values. This is because fluorescent probe $\mathbf{C}$ has sensitive $\mathrm{pH}$ responses (14.3-fold enhancement from $\mathrm{pH}$ 3.02 to $\mathrm{pH} 9.49$ ), $\mathrm{p} K_{\mathrm{a}}$ value near physiological $\mathrm{pH}$, deep-red emission that prevents the cell damage, and large Stokes shift (115 nm in buffer solution at $\mathrm{pH}$ 7.4) that minimizes the potential interference caused by excitation signals. It should be noted that many reported morpholine contained fluorescent probes and dyes show labeling of lysosomes and other acidic organelles in mammalian cells due to their accumulative effect of tertiary amines on the morpholine moieties in acidic condition. ${ }^{9}$ Therefore, to investigate whether our probe could respond to intracellular $\mathrm{pH}$ by identifying these inherently acid compartments and the corresponding probe concentration effect, we first incubated HUVEC-C cells with $5 \mu \mathrm{M}, 15$ $\mu \mathrm{M}$ and $25 \mu \mathrm{M}$ fluorescent probe $\mathbf{C}$ and compared with a well-known commercial lysosome probe, LysoSensor Green DND-189 that specifically labels the acidic organelles in cells. The results show that probe $\mathbf{C}$ exhibited very weak fluorescence signals in all three concentrations although slight fluorescence enhancements could be observed with increased probe concentrations (Figure 5). The merged fluorescence images shows green-yellowish areas around nucleus and many green-yellowish dot structures also can be observed by a careful examination (Figure 5). The calculated Pearson's coefficients of red (fluorescent probe $\mathbf{C}$ ) and green (LysoSensor Green) channels are $0.92,0.85$ and 0.88 for $5 \mu \mathrm{M}, 15 \mu \mathrm{M}$ and $25 \mu \mathrm{M}$ probe $\mathbf{C}$, respectively (please see Figures S15-S17 in supporting information for cytofluorograms). The results indicate the area stained by the fluorescent probe $\mathbf{C}$ matches those stained by LysoSensor Green DND-189 in cells, which confirms fluorescent probe $\mathbf{C}$ mainly stained lysosomes or other acidic organelles in cells (Figure 5). Therefore, the weak fluorescence of probe $\mathbf{C}$ observed in the cells is able to be elucidated via a d-PET modulated fluorescence quenching by the protonated morpholine moieties under lysosomal $\mathrm{pH}$ (4.5-5.5), which demon-strates the ability of $\mathrm{pH}$ response for probe $\mathbf{C}$ to the inherent acidic environment in live cells. In order to examine the fluorescence responses of fluorescent probe $\mathbf{C}$ to different $\mathrm{pH}$ inside of cells, we further incubated HUVEC-C cells with $5 \mu \mathrm{M}, 15$ $\mu \mathrm{M}$ and $25 \mu \mathrm{M}$ fluorescent probe $\mathbf{C}$ in buffer solutions ( $\mathrm{K}^{+}$rich PBS) at $\mathrm{pH}$ 5.5, 6.5, 7.5, or 8.5 having nigericin $\left(\mathrm{H}^{+} / \mathrm{K}^{+}\right.$ionophore). Nigericin is a widely used ionophore for adjusting the intracellular $\mathrm{pH}\left(\mathrm{pH}_{\mathrm{i}}\right)$ through equilibrating the intracellular and extracellular $\mathrm{pHs}$. At all three concentrations, fluorescent probe $\mathbf{C}$ displayed very weak fluorescence at acidic $\mathrm{pH}(\mathrm{pH}$ 5.5), whereas its fluorescence intensity showed gradual enhancement as intracellular $\mathrm{pH}$ increased from 5.5 to 8.5 (Figure 6). In addition, at each intracellular $\mathrm{pH}$, higher probe concentration resulted in stronger fluorescence signals (Figure 6). These responses of fluorescent probe $\mathbf{C}$ to intracellular $\mathrm{pH}$ are in line with its optical responses to $\mathrm{pH}$ in buffer solutions (Figure 2), which further proved the d-PET mechanism of fluorescent probe $\mathbf{C}$ at different $\mathrm{pH}$ in live cells. However, the commercial lysosome probe LysoSensor Green DND-189 did not exhibit any change in fluorescence upon change of intracellular $\mathrm{pH}$ (please see Figures S18-S20 in supporting information).

We also investigated the toxicity of fluorescent probe $\mathbf{C}$ to HUVEC-C cells using MTS assay (Figure 7). At low concentration of $5 \mu \mathrm{M}$, the fluorescent probe $\mathbf{C}$ provided more than $80 \%$ 
cell viability, when the concentration increased to $15 \mu \mathrm{M}$, the cell viability dropped to 60-70\%, indicating the low to moderate toxicity of fluorescent probe $\mathbf{C}$ to the cells in this concentration range. However, fluorescent probe $\mathbf{C}$ at higher concentrations like $25 \mu \mathrm{M}$ and $50 \mu \mathrm{M}$ were very toxic to the cells as less than $10 \%$ cell viability were observed, which may limit the application of the probe $\mathbf{C}$ with this high concentrations, and the reason for this toxicity is still under investigation. However, we are still able to effectively image and visualize $\mathrm{pH}$ changes by using low concentration of probe $\mathbf{C}$ (with less than $15 \mu \mathrm{M}$ ) with the relative low cellular toxicity.

\section{Conclusion}

We have successfully prepared three $\mathrm{pH}$ sensitive morpholine-functionalized fluorescent probes $\mathbf{A}, \mathbf{B}$ and $\mathbf{C}$. These probes display unusual $\mathrm{pH}$ responses in aqueous solutions with high fluorescence in basic conditions while their fluorescence get quenched in acidic condition due to the d-PET effect from protonated morpholine moieties to the BODIPY cores. All three probes are photostable and display selectively responses to $\mathrm{pH}$ over common metal ions. Fluorescent probe $\mathbf{C}$ provided a potential non-invasive method with deep-red fluorescence and low background for monitoring intracellular $\mathrm{pH}$ changes inside of living cells.

\section{Supplementary Material}

Refer to Web version on PubMed Central for supplementary material.

\section{Acknowledgments}

Funding Sources

This research reported in this publication was supported by the National Institute of General Medical Sciences of the National Institutes of Health under Award Number R15GM114751 (to H.Y. Liu and A. Tiwari). The research was also partially supported by the National Science Foundation (award number 1048655) (to H. Y. Liu), Michigan Technological University faculty start-up fund, and Research Excellence Fund (to A. Tiwari).

\section{REFERENCES}

(1). Han JY, Burgess K. Fluorescent Indicators for Intracellular pH. Chem. Rev. 2010; 110:2709-2728. [PubMed: 19831417]

(2). Fan JL, Dong HJ, Hu MM, Wang JY, Zhang H, Zhu H, Sun W, Peng XJ. Fluorescence Imaging Lysosomal Changes During Cell Division and Apoptosis Observed Using Nile Blue Based Nearinfrared Emission. Chem. Commun. 2014; 50:882-884.

(3). Fan JL, Lin CY, Li HL, Zhan P, Wang JY, Cui S, Hu MM, Cheng GH, Peng XJ. A Ratiometric Lysosomal pH Chemosensor Based on Fluorescence Resonance Energy Transfer. Dyes Pigm. 2013; 99:620-626.

(4). Zhang H, Fan JL, Dong HJ, Zhang SZ, Xu WY, Wang JY, Gao P, Peng XJ. Fluorene-derived Twophoton Fluorescent Probes for Specific and Simultaneous Bioimaging of Endoplasmic Reticulum and Lysosomes: Group-effect and Localization. J. Mater. Chem. B. 2013; 1:5450-5455.

(5). zhang XF, Wang C, Han Z, Xiao Y. A Photostable Near-Infrared Fluorescent Tracker with pHIndependent Specificity to Lysosomes for Long Time and Multicolor Imaging. ACS Appl. Mater. Interfaces. 2014; 6:21669-21676. [PubMed: 25382852]

(6). Wang L, Xiao Y, Tian WM, Deng LZ. Activatable Rotor for Quantifying Lysosomal Viscosity in Living Cells. J. Am. Chem. Soc. 2013; 135:2903-2906. [PubMed: 23409947] 
(7). Vegesna GK, Janjanam J, Bi JH, Luo FT, Zhang JT, Olds C, Tiwari A, Liu HY. pH-Activatable Near-infrared Fluorescent Probes for Detection of Lysosomal pH Inside Living Cells. J. Mater. Chem. B. 2014; 2:4500-4508.

(8). Zhang JT, Yang M, Li C, Dorh N, Xie F, Luo FT, Tiwari A, Liu HY. Near-infrared Fluorescent Probes Based on Piperazine-functionalized BODIPY Dyes for Sensitive Detection of Lysosomal pH. J. Mater. Chem. B. 2015; 3:2173-2184.

(9). Galindo F, Burguete MI, Vigara L, Luis SV, Kabir N, Gavrilovic J, Russell DA. Synthetic Macrocyclic Peptidomimetics as Tunable $\mathrm{pH}$ Probes for The Fluorescence Imaging of Acidic Organelles in Live Cells. Angew. Chem.-Int. Edit. 2005; 44:6504-6508.

(10). Chauhan DP, Varma SJ, Vijeta A, Banerjee P, Talukdar P. A 1,3-Amino Group Migration Route to form Acrylamidines. Chem. Commun. 2014; 50:323-325.

(11). Becke AD. Density-functional Thermochemistry .3. The Role of Exact Exchange. J. Chem. Phys. 1993; 98:5648-5652.

(12). Becke AD. A New Mixing of Hartree-fock and Local Density-functional Theories. J. Chem. Phys. 1993; 98:1372-1377.

(13). Lee CT, Yang WT, Parr RG. Development of The Colle-salvetti Correlation-energy Formula into A Functional of The Electron-density. Phys. Rev. B: Condens. Matter Mater. Phys. 1988; 37:785789.

(14). Hehre, WJ.; Radom, L.; Schelyer, PVR.; Pople, JA. Ab Initio Molecular Orbital Theory. Wiley; New York: 1986.

(15). Frisch, MJ.; Trucks, GW.; Schlegel, HB.; Scuseria, GE.; Robb, MA.; Cheeseman, JR.; Scalmani, G.; Barone, V.; Mennucci, B.; Petersson, GA., et al. Gaussian 09. Gaussian, Inc.; Wallingford CT: 2009.

(16). Bolte S, Cordelieres FP. A Guided Tour into Subcellular Colocalization Analysis in Light Microscopy. J. Microsc. (Oxford, U. K.). 2006; 224:213-232.

(17). Zhu SL, Zhang JT, Vegesna GK, Pandey R, Luo FT, Green SA, Liu HY. One-pot Efficient Synthesis of Dimeric, Trimeric, and Tetrameric BODIPY Dyes for Panchromatic Absorption. Chem. Commun. 2011; 47:3508-3510.

(18). Zhu SL, Zhang JT, Vegesna G, Tiwari A, Luo FT, Zeller M, Luck R, Li HH, Green S, Liu HY. Controlled Knoevenagel Reactions of Methyl Groups of 1,3,5,7-Tetramethyl BODIPY Dyes for Unique BODIPY Dyes. RSC Adv. 2012; 2:404-407.

(19). Zhu SL, Bi JH, Vegesna G, Zhang JT, Luo FT, Valenzano L, Liu HY. Functionalization of BODIPY Dyes at 2,6-Positions through Formyl Groups. RSC Adv. 2013; 3:4793-4800. 

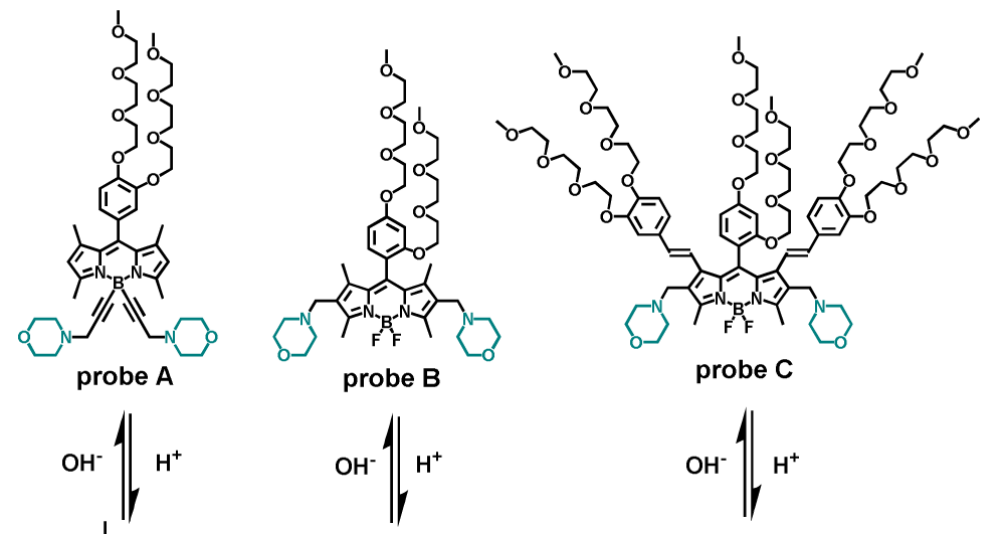

$\mathrm{OH}^{-} \| \mathrm{H}^{+}$

$\mathrm{OH}^{-} \| \mathrm{H}^{+}$
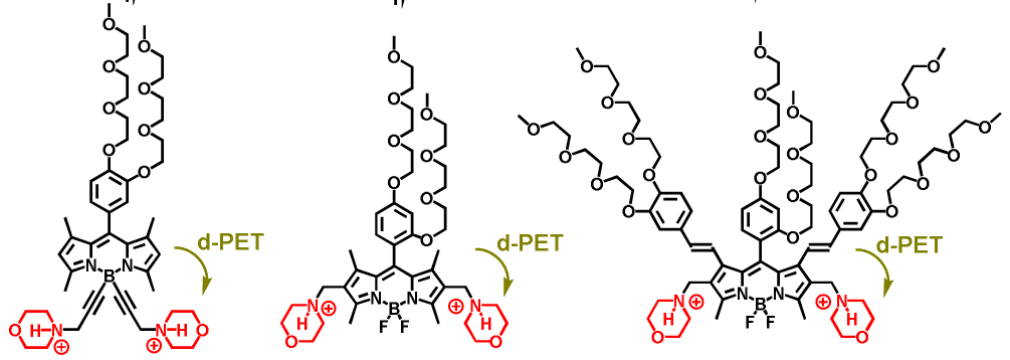

Figure 1.

Chemical structures of fluorescent probes $\mathbf{A}, \mathbf{B}$ and $\mathbf{C}$ and their responses to acid and base. 

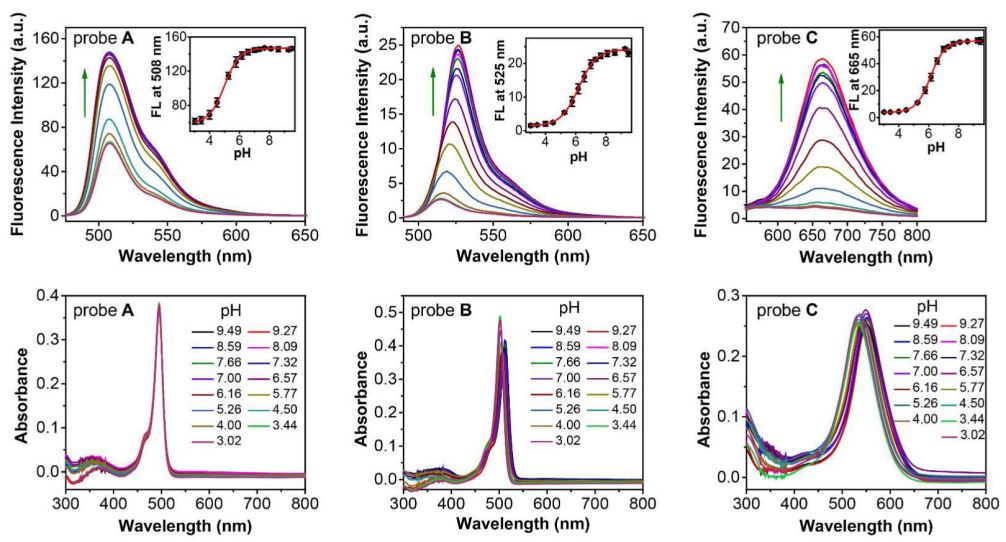

Figure 2.

Fluorescence (upper row) and absorption spectra (lower row) of fluorescent probes A, B and $\mathbf{C}(5 \mu \mathrm{M})$ in buffer solution with different $\mathrm{pH}$ conditions (1\% DMSO as co-solvent). Insets in upper row: the corresponding curves of fluorescence intensity versus $\mathrm{pH}$. 


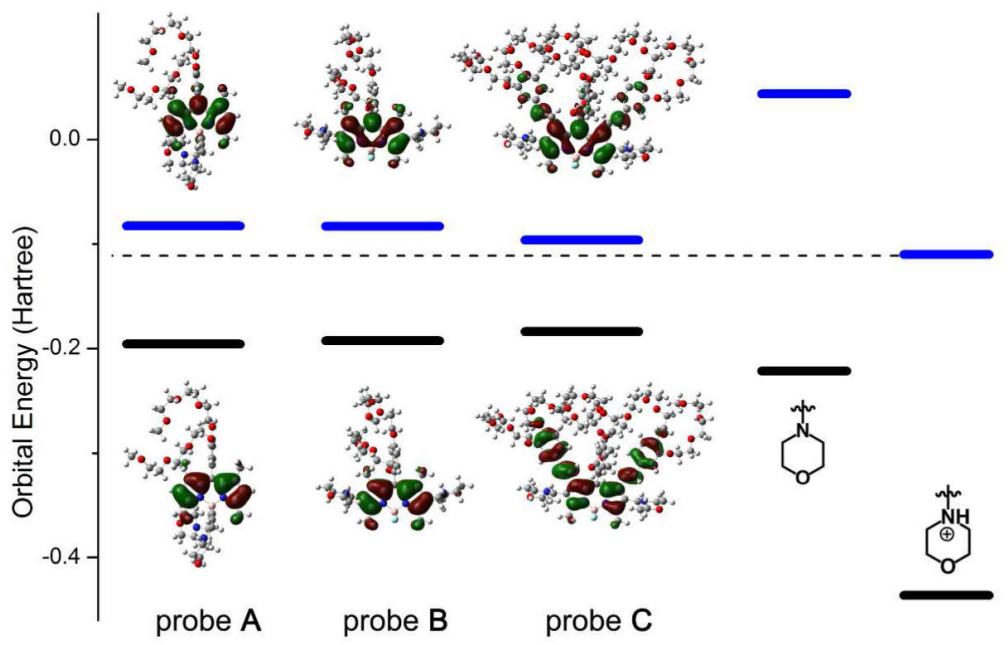

Figure 3.

Comparisons of calculated HOMO and LUMO energies of fluorescent probes $\mathbf{A}, \mathbf{B}$ and $\mathbf{C}$, morpholine moiety ( $\mathrm{N}$-methylmorpholine) and protonated morpholine moiety (protonated $N$-methylmorpholine), as well as the electron density distributions in HOMO and LUMO of fluorescent probes $\mathbf{A}, \mathbf{B}$ and $\mathbf{C}$. 

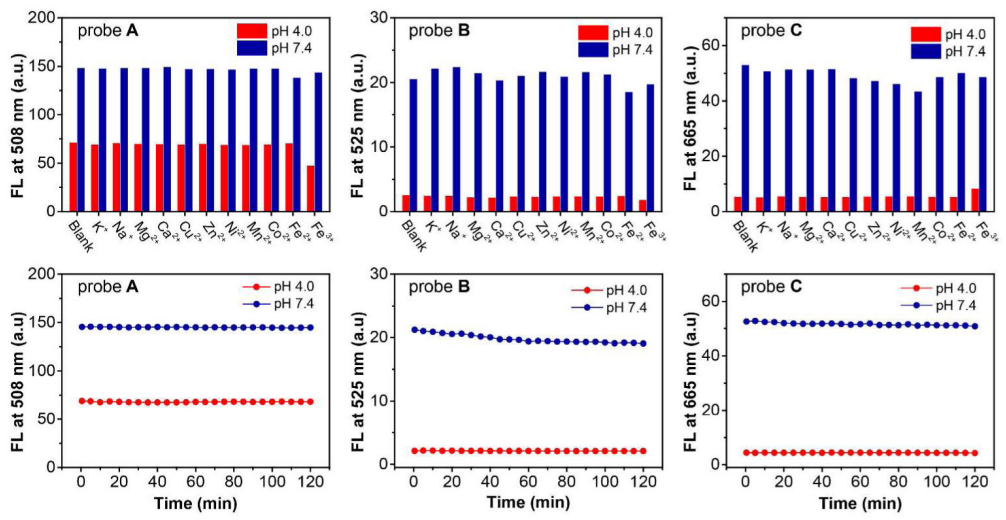

Figure 4.

Upper row: fluorescence responses of fluorescent probes $\mathbf{A}, \mathbf{B}$ and $\mathbf{C}(5 \mu \mathrm{M})$ to $\mathrm{pH}$ at 4.0 and 7.4 in the absence and presence of various metal ions $(200 \mu \mathrm{M})$ in buffer solutions (with $1 \%$ DMSO as co-solvent). Lower row: fluorescence intensities changes of fluorescent probes $\mathbf{A}, \mathbf{B}$ and $\mathbf{C}(5 \mu \mathrm{M})$ as a function of time in 2 hours under excitation in buffer solutions (1\% DMSO as co-solvent) at $\mathrm{pH} 4.0$ and 7.4. 


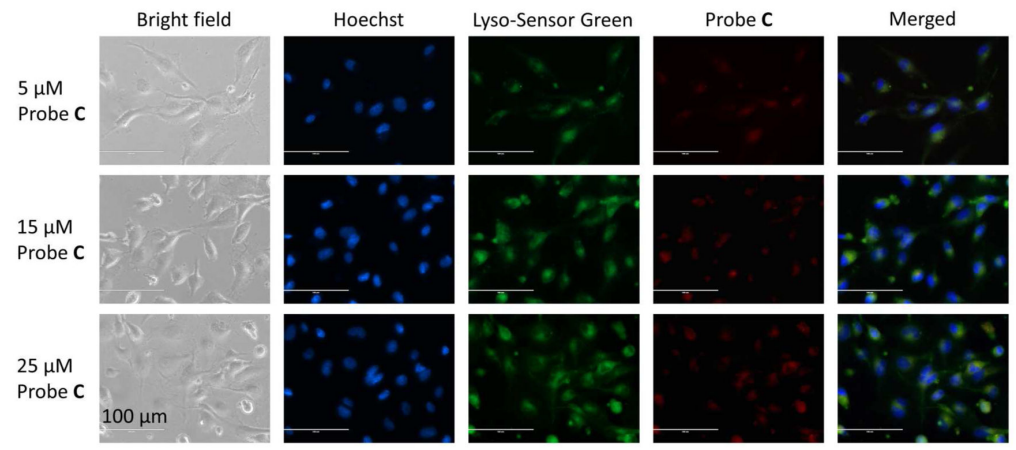

Figure 5.

Fluorescence images of HUVEC-C cells incubated with $5 \mu \mathrm{M}, 15 \mu \mathrm{M}$, or $25 \mu \mathrm{M}$ fluorescent probe $\mathbf{C}$. HUVEC-C cells were incubated with fluorescent probe $\mathbf{C}$ for $2 \mathrm{~h}$, post serum starvation $(2 \mathrm{~h})$ and imaged for co-localization with $1 \mu \mathrm{M}$ LysoSensor Green and (1 $\mu \mathrm{g} \cdot \mathrm{mL}^{-1}$ ) Hoechst 33342 stains. Images were acquired using the inverted fluorescence microscope (AMF-4306, EVOS $\left._{\mathrm{fl}}, \mathrm{AMG}\right)$ at $40 \times$ magnification, scale bars $=100 \mu \mathrm{m}$. 


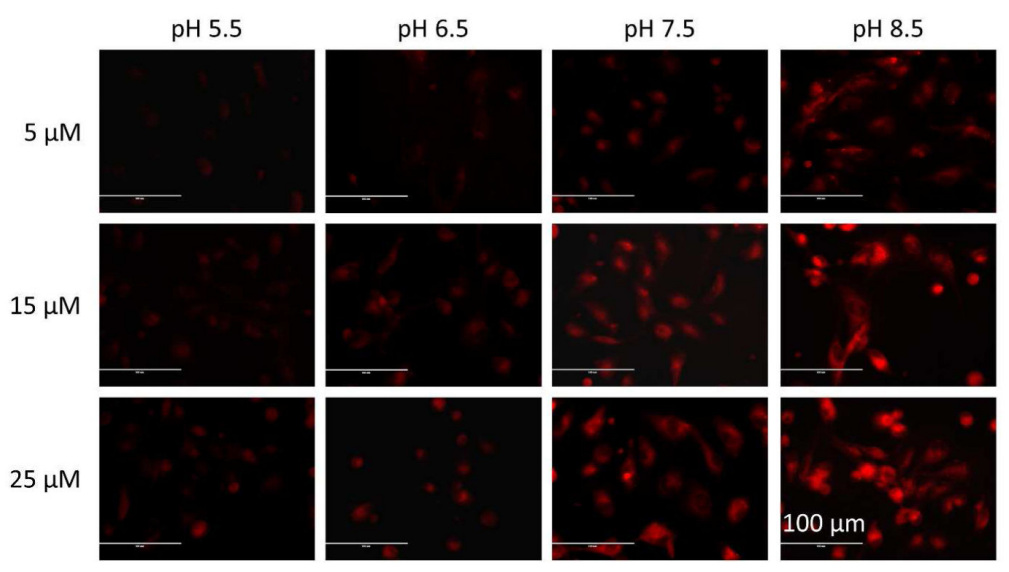

Figure 6.

Fluorescence images of HUVEC-C cells incubated with $5 \mu \mathrm{M}, 15 \mu \mathrm{M}$, or $25 \mu \mathrm{M}$ fluorescent probe $\mathbf{C}$ in buffers at different $\mathrm{pH}$ values of $5.5,6.5,7.5$, or 8.5 having nigericin. Images were acquired using the inverted fluorescence microscope (AMF-4306, EVOSfl, AMG) at $40 \times$ magnification, scale bars $=100 \mu \mathrm{m}$. 


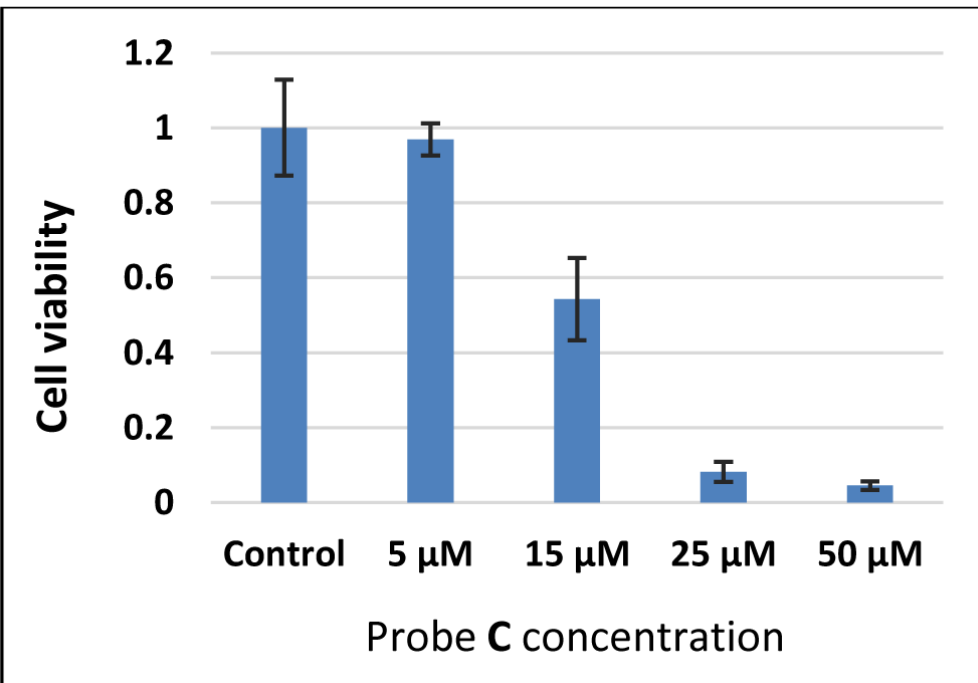

Figure 7.

Effects of fluorescent probe $\mathbf{C}$ on cell proliferation measured by MTS assay. HUVEC-C cells were incubated with $5 \mu \mathrm{M}, 15 \mu \mathrm{M}, 25 \mu \mathrm{M}$, or $50 \mu \mathrm{M}$ of fluorescent probe $\mathbf{C}$ for $48 \mathrm{~h}$. To this $20 \mu \mathrm{L}$ of MTS reagent was added per well and absorbance at $490 \mathrm{~nm}$ was measured to determine cell viability. Error bars indicate \pm SD. 


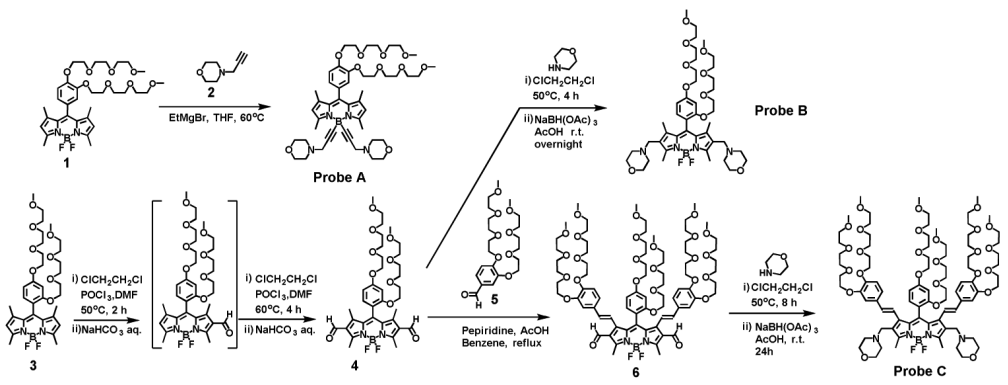

Scheme 1.

Synthetic route to fluorescent probes $\mathbf{A}, \mathbf{B}$ and $\mathbf{C}$. 